\title{
Óleo essencial de aroeira-vermelha como aditivo na ração de frangos de corte
}

\author{
Essential oil from Brazillian red pepper as an additive in broiler diet
}

\author{
Maria Aparecida da Silva ${ }^{\mathrm{I}}$ Bruna Mirelly de Sousa Pessotti ${ }^{\mathrm{I}}$ Surama Freitas Zanini ${ }^{\mathrm{*}}$ \\ Geraldo Luiz Colnago ${ }^{\text {II }}$ Louisiane de Carvalho Nunes ${ }^{\mathrm{I}}$ Maria Regina Alves Rodrigues ${ }^{\mathrm{II}}$ \\ Larissa Ferreira ${ }^{\mathrm{I}}$
}

\section{RESUMO}

\begin{abstract}
Objetivou-se avaliar os efeitos da inclusão de óleo essencial de aroeira-vermelha (Schinus terebinthifolius Raddi) como promotor de crescimento nas rações de frangos de corte sobre o desempenho e morfometria intestinal desses animais. Foram utilizados 300 pintos de um dia de vida, tipo corte, machos, linhagem Cobb. As aves foram alojadas em 20 boxes de $2 \mathrm{~m}^{2}$ (15 aves boxe $\left.{ }^{-1}\right)$, distribuídos em um delineamento inteiramente casualizado, em grupos de quatro (4) tratamentos e cinco (5) repetições, com 15 aves cada. Os grupos foram identificados como T1: dieta sem promotor de crescimento e sem óleo de aroeira-vermelha (controle negativo); T2: dieta com promotor de crescimento e sem óleo de aroeira (antimicrobiano e anticoccidiano - controle positivo); T3: dieta contendo somente antimicrobiano (bacitracina de zinco); T4: dieta com $0,4 \%$ de óleo de aroeira-vermelha. Não houve efeito significativo dos tratamentos sobre o desempenho animal $(P>0,05)$. Contudo, quanto aos aspectos morfométricos dos intestinos verificou-se que, aos 21 dias de idade, os animais tratados com promotor de crescimento apresentaram maior relação vilo:cripta, que não diferiu dos tratados com 0,4\% de óleo de aroeira-vermelha $(P>0,05)$. As aves arraçoadas com uma dieta sem promotor de crescimento (sem antibiótico e anticoccidiano) tiveram a menor relação vilo:cripta $(P<0,05)$. $A$ adição de óleo de aroeira-vermelha na dieta de frangos de corte reduziu o peso relativo dos intestinos quando comparado com a dieta sem promotor de crescimento $(P<0,05)$, não diferindo dos tratados com promotor de crescimento $(P>0,05)$. Neste estudo, não houve diferença significativa na profundidade das criptas de Lieberkühn e na altura das vilosidades entre os tratamentos (P>0,05). Concluiu-se que a adição de 0,4\% de óleo de aroeira promoveu uma melhoria na superfície absortiva intestinal das aves, quando comparado com as aves alimentadas sem promotor de crescimento.
\end{abstract}

Palavras-chave: avicultura, Schinus terebinthifolius Raddi, desempenho, morfometria intestinal, promotor de crescimento.

\section{ABSTRACT}

The aim was to evaluate the effect of inclusion of Brazilian red pepper oil (Schinus terebinthifolius Raddi), as an additive in feed on performance and intestinal morphometry of broilers. A total of 300 day-old male chicks, Cobb, distributed in a randomized design in groups of four (4) treatments and five repetitions, with 15 birds each: diet without growth promoter and without Brazilian red pepper oil (negative control); diet with growth promoter (antimicrobial and anticoccidial - positive control); diet containing only antimicrobial (bacitracin zinc); diet with $0.4 \%$ of Brazilian red pepper oil. It was not verified significantly dietary effects on the performance of birds (P>0.05). At 21 days of age, the broilers chicks fed with growth promoter showed greater ratio of villous:crypt which not differs from chickens fed with $0.4 \%$ Brazilian red pepper oil $(P<0.05)$. The diet without growth promoter resulted in the lowest ratio of villous:crypt $(P<0.05)$. The addition of Brazilian red pepper in the diet of broiler chickens reduced the relative weight of the intestines compared to broilers fed diet without growth promoter $(P<0.05)$. In this study there was no significant difference in crypt depth and in villous height between treatments ( $P>0.05)$. It was concluded that the addition of $0.4 \%$ Brazilian red pepper oil resulted an improvement in intestinal absorptive surface of broilers compared with chickens fed with diet without growth promoter.

Key words: aviculture, Schinus terebinthifolius Raddi, essential oil, intestinal morphometry, performance, growth promoter.

'Departamento de Medicina Veterinária, Centro de Ciências Agrárias, Universidade Federal do Espírito Santo (UFES), Alto Universitário s/n, CP 16, 29500-000, Alegre, ES, Brasil. E-mail: surama@cca.ufes.br. *Autor para correspondência.

II Departamento de Zootecnia, Universidade Federal Fluminense (UFF), Niterói, RJ, Brasil.

IIIDepartamento de Química Orgânica, Universidade Federal de Pelotas (UFPEL), Pelotas, RS, Brasil. 


\section{INTRODUÇÃO}

Com a proibição do uso de antibióticos como promotores de crescimento, torna-se imprescindível o uso de ferramentas alternativas que podem ser usadas sem suscitar o risco de resistência bacteriana (CORNELI, 2004). De acordo com MENTEN (2002), uma classe de produtos que pode vir a substituir os agentes antimicrobianos consiste dos aditivos fitogênicos, extratos herbais ou extratos vegetais.

Estudos fitoquímicos identificaram fenóis, flavonóides, esteróides, triterpenos, antraquinonas e saponinas em plantas da espécie Schinus terebinthifolius Raddi (MELO JÚNIOR et al., 2000). Atividades antiinflamatória (GAZZANEO et al., 2005) e antimicrobiana (MARTINEZ et al., 1996; SCHMOURLO et al., 2005; LIMA et al., 2006a; LIMA et al., 2006b) têm sido demonstradas pelo uso dessa planta em diferentes modelos farmacológicos. Seu óleo essencial não mostrou nenhuma toxicidade em animais e humanos (BARBOSA et al., 2007). Objetivou-se avaliar a eficácia do óleo essencial de aroeira-vermelha (S. terebinthifolius Raddi) como aditivo na ração de frangos de corte sobre seu desempenho e morfometria intestinal.

\section{MATERIAL E MÉTODOS}

O experimento foi conduzido no Setor de Avicultura da Escola Agrotécnica Federal de Alegre, localizada no Distrito de Rive. As avaliações morfométricas foram realizadas no Laboratório de Anatomia Patológica Veterinária do Centro de Ciências Agrárias da Universidade Federal do Espírito Santo (CCA-UFES).

Foram utilizados 300 pintos de um dia, de corte, machos, da linhagem Cobb, alojados em 20 boxes, que possuíam cama nova, medindo $2 \mathrm{~m}^{2}\left(15\right.$ aves boxe $\left.{ }^{-1}\right)$, distribuídos em um delineamento inteiramente casualizado, em grupos de quatro (4) tratamentos e cinco (5) repetições, com 15 aves cada: T1: dieta sem promotor de crescimento e sem óleo de aroeiravermelha (controle negativo); T2: dieta com promotor de crescimento e sem óleo de aroeira (antimicrobiano e anticoccidiano - controle positivo); T3: dieta contendo somente antimicrobiano (bacitracina de zinco); $\mathrm{T} 4$ : dieta com $0,4 \%$ de óleo de aroeira-vermelha, nível padronizado previamente por experimento pilo. No período de 1 a 21 dias de idade, os animais foram criados com uma ração inicial ad libitum para frangos de corte com $22 \%$ de PB e $2900 \mathrm{Kcal} \mathrm{EM} \mathrm{kg}^{-1}$. Do $22^{\circ}$ ao $38^{\circ}$ dia de idade, as aves foram alimentadas com a ração $a d$ libitum de crescimento com $19 \%$ PB e $3000 \mathrm{kcal} \mathrm{EM} \mathrm{kg}^{-1}$ à base de milho e soja. A bacitracina de zinco $15 \%$ $\left(45 \mathrm{mg} \mathrm{kg}^{-1}\right)$ e a salinomicina $12 \%\left(66 \mathrm{mg} \mathrm{kg}^{-1}\right)$ foram o antibiótico e o anticoccidiano, respectivamente, utilizados na dieta do grupo controle positivo.

$\mathrm{O}$ óleo essencial de frutos maduros de aroeira-vermelha (Schinus terebinthifolius Raddi) foi obtido pela técnica de destilação por arraste a vapor, de acordo com BERTOLDI (2006). As amostras do óleo essencial de aroeira-vermelha foram submetidas à análise por Cromatografia Gasosa acoplado com Espectrometria de Massa (CG-MS), em um equipamento GC-MS (Schimadzu, modelo 5050 A) equipado com uma

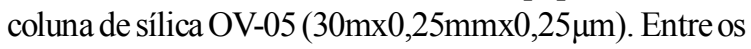
compostos presentes no óleo essencial de aroeiravermelha, obtido de frutos maduros, o $\alpha$-pineno foi o constituinte majoritário $(29,39 \%)$, seguido pelo $\delta$ - careno (19,69\%), limoneno (18,15\%) e $\alpha$-felandreno $(9,39 \%)$.

As aves foram pesadas aos 19 e 38 dias de idade, visando determinação do ganho de peso. No cálculo do consumo de ração, consideraram-se a ração fornecida, os desperdícios e as sobras nos comedouros, ocorridos durante o período experimental. Assim, o consumo de ração foi obtido com base no consumo médio por unidade experimental dentro de cada fase. Os dados de conversão alimentar foram obtidos com base no ganho de peso e no consumo médio de ração por fase.

No $22^{\circ}$ e $38^{\circ}$ dias de idade, quatro aves por tratamento foram escolhidas de acordo com o peso médio do boxe e abatidas por meio de deslocamento cervical para coleta de fragmentos de jejuno com quatro centímetros de comprimento. Esse material foi imediatamente lavado em solução fisiológica e fixado em solução de Bouin. Os fragmentos foram submetidos ao processo de inclusão em parafina, de acordo com a técnica histopatológica de rotina. Secções com espessura de $7 \mu \mathrm{m}$ foram corados com hematoxilinaeosina. Foram feitas mensurações no jejuno, de acordo com MAIORKA et al. (2000), da altura da vilosidade intestinal, desde o epitélio luminal até a camada muscular da mucosa e da profundidade da cripta, medida do epitélio luminal da cripta até a muscular da mucosa e a relação vilo:cripta. A morfometria do jejuno foi realizada utilizando-se o aumento de 100 vezes (objetiva 10x). A captura de imagem foi feita com Câmera Olympus Polaroid e sua análise foi feita a partir do software Sigma Scan Pro 5.0.

As análises estatísticas dos parâmetros avaliados foram realizadas com utilização do programa SAEG (Sistema para Análises Estatísticas e Genéticas). Os resultados foram submetidos à análise de variância 
utilizando o teste Tukey para comparação de médias entre os tratamentos $(\mathrm{P}<0,05)$.

\section{RESULTADOS E DISCUSSÃO}

O frango de corte está sujeito a diversos fatores capazes de alterar as características morfofuncionais da mucosa gastrintestinal, como as lesões microbianas, que podem interferir no turnover celular e consequentemente no processo absortivo de nutrientes (MACARI et al., 1994). Ao analisar o desempenho animal, não foi verificado o efeito significativo dos tratamentos sobre os índices de produção animal (Tabela 1, P>0,05). Esses resultados indicam que as condições experimentais não permitiram observar o efeito dos tratamentos, já que o grupo tratado com antibiótico não diferiu dos não tratados. De acordo com CROMWELL (1991) e MENTEN (2001), o efeito benéfico dos antibióticos é maior em condições de campo, com respostas duas vezes maiores que as observadas em estações experimentais, por causa das diferenças de higiene e estresse e pela presença de doenças. FREITAS et al. (2001) não registraram diferenças significativas no desempenho de frangos aos 24 dias recebendo dietas com extrato vegetal ou antibiótico, e atribuíram os resultados encontrados ao baixo desafio sanitário que as aves estavam expostas. Também não foram observadas diferenças significativas com o uso de outros promotores de crescimento, como os prebióticos e probióticos, em substituição ao antibiótico (ALVAREZ et al., 1994;
CAVALCANTI et al., 1996; HENRIQUE et al., 2000; MOREIRA et al., 2001)

Aos 21 dias de idade, os animais tratados com promotores de crescimento apresentaram maior relação vilo:cripta, que não diferiu dos tratados com $0,4 \%$ de óleo de aroeira-vermelha (Tabela $2, \mathrm{P}<0,05$ ). As aves arraçoadas com uma dieta sem promotor de crescimento (sem antibiótico e anticoccidiano) tiveram a menor relação vilo:cripta $(\mathrm{P}<0,05)$, semelhante ao encontrado por TSINAS (2003) e SILVA et al. (2009) ao utilizarem a adição de óleo de orégano na dieta de frangos de corte. Discordando de FUKAYAMA (2004), que não observou diferença ao realizar seu estudo da morfometria intestinal de frangos de corte alimentados com óleo de orégano, e de BALOG NETO et al. (2007), que ao realizarem a morfometria de frangos de corte que tiveram a utilização de simbióticos na dieta também não observaram melhora na morfometria intestinal. Porém, este resultado não foi suficiente para levar a uma diferença no desempenho das aves.

A relação vilo: cripta é um indicador da capacidade digestiva do intestino delgado. De acordo com LUQUETTI (2005), uma relação vilo: cripta diminuída indica vilos danificados e uma atividade proliferativa aumentada nas criptas, objetivando restaurar a forma e a função do epitélio. Por outro lado, um aumento nessa taxa corresponde a um aumento na digestão e absorção de nutrientes (MONTAGNE et al., 2003). Neste estudo, não houve diferença significativa na profundidade das criptas de Lieberkühn e na altura dos vilos entre os tratamentos $(\mathrm{P}>0,05)$.

Tabela 1 - Consumo de ração ( $\mathrm{g}$ ave $\mathrm{e}^{-1}$ ), ganho de peso $\left(\mathrm{g}^{\mathrm{ave}} \mathrm{e}^{-1}\right)$ e conversão alimentar dos frangos de corte alimentados com dieta não contendo nem antibiótico nem anticoccidiano, contendo antibiótico e anticoccidiano, contendo somente antibiótico e com óleo de aroeira a $0,4 \%$ durante o período de 1-19 e 1-38 dias.

\begin{tabular}{|c|c|c|}
\hline \multirow{2}{*}{ Dietas } & 1-19 dias & $1-38$ dias \\
\hline & 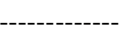 & ------- \\
\hline Controle negativo (sem antibiótico e anticoccidiano) & 892,8 & 2769,76 \\
\hline Controle positivo (com antibiótico e anticoccidiano) & 892,8 & 2734,91 \\
\hline Somente com antibiótico & 943,0 & 2760,03 \\
\hline $0,4 \%$ óleo de aroeira-vermelha & 934,0 & 2620,54 \\
\hline Controle negativo (sem antibiótico e anticoccidiano) & 574,33 & 1426,05 \\
\hline Controle positivo (com antibiótico e anticoccidiano) & 574,64 & 1474,90 \\
\hline Somente com antibiótico & 586,54 & 1503,03 \\
\hline $0,4 \%$ óleo de aroeira-vermelha & 555,94 & 1389,13 \\
\hline Controle negativo (sem antibiótico e anticoccidiano) & 1,58 & 1,95 \\
\hline Controle positivo (com antibiótico e anticoccidiano) & 1,56 & 1,83 \\
\hline Somente com antibiótico & 1,62 & 1,83 \\
\hline $0,4 \%$ óleo de aroeira-vermelha & 1,70 & 1,87 \\
\hline
\end{tabular}

Ciência Rural, v.41, n.4, abr, 2011. 
Tabela 2 - Valores observados e expressos em micrômetros da morfometria da altura das vilosidades, criptas de Lieberkühn e da relação vilo:cripta de frangos de corte de 15 e 21 dias tratados com óleo de aroeira vermelha ou com promotor de crescimento (antibiótico e anticoccidiano).

\begin{tabular}{|c|c|c|}
\hline \multirow{2}{*}{ Dietas } & $15^{\circ}$ dia de idade & $21^{\circ}$ dia de idade \\
\hline & \multicolumn{2}{|c|}{ 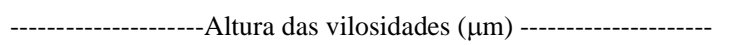 } \\
\hline Controle negativo (sem antibiótico e anticoccidiano) & 1033,54 & 1456,35 \\
\hline Controle positivo (com antibiótico e anticoccidiano) & 1133,30 & 1507,98 \\
\hline Somente com antibiótico & 1144,03 & 1381,61 \\
\hline \multirow[t]{2}{*}{$0,4 \%$ óleo de aroeira-vermelha } & 1099,59 & 1361,45 \\
\hline & \multicolumn{2}{|c|}{---------Profundidade das criptas de Lieberkühn $(\mu \mathrm{m})$------ } \\
\hline Controle negativo (sem antibiótico e anticoccidiano) & 136,53 & 261,63 \\
\hline Controle positivo (com antibiótico e anticoccidiano) & 169,30 & 209,04 \\
\hline Somente com antibiótico & 175,28 & 232,98 \\
\hline \multirow[t]{2}{*}{$0,4 \%$ óleo de aroeira-vermelha } & 149,68 & 229,80 \\
\hline & \multicolumn{2}{|c|}{ 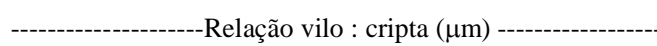 } \\
\hline Controle negativo (sem antibiótico e anticoccidiano) & 7,66 & $5,84 \mathrm{~B}$ \\
\hline Controle positivo (com antibiótico e anticoccidiano) & 6,78 & $7,22 \mathrm{~A}$ \\
\hline Somente com antibiótico & 6,72 & $5,95 \mathrm{AB}$ \\
\hline $0,4 \%$ óleo de aroeira-vermelha & 7,43 & $5,98 \mathrm{AB}$ \\
\hline
\end{tabular}

${ }^{\mathrm{A}, \mathrm{B}}$ Médias seguidas por letras distintas na mesma coluna diferem significativamente pelo teste Tukey $(\mathrm{P}<0,05)$.

Os dados referentes ao peso relativo dos intestinos (delgado e ceco) encontram-se na tabela 3. Aos 15 dias de idade, verificou-se que a adição de óleo de aroeira-vermelha na dieta de frangos de corte reduziu o peso relativo dos intestinos, quando comparado com a dieta sem promotor de crescimento, não diferindo dos tratados com promotor de crescimento (Tabela 3; $\mathrm{P}<0,05)$. COATES et al. (1963) relataram que os promotores de crescimento podem atuar como agentes modificadores, diminuindo a espessura da parede intestinal devido à eliminação de bactérias danosas. Segundo POLLMAN (1986), a presença de microrganismos patogênicos provoca lesões e espessamento das paredes do trato gastrintestinal, possivelmente decorrente da ação de toxinas que são liberadas nesses locais. Adicionalmente, LODDI et al. (2000) relatou que um espessamento da parede intestinal pode prejudicar a absorção e diminuir a quantidade de nutrientes disponíveis ao animal.

\section{CONCLUSÃO}

A adição de $0,4 \%$ de óleo de aroeira promove uma melhoria na superfície absortiva intestinal e proprociona uma diminuição no peso relativo dos intestinos delgado e grosso das aves, quando comparado com as aves alimentadas sem promotor de crescimento. Ao se observar o desempenho animal, a adição de $0,4 \%$ de óleo de aroeira gerou o mesmo ganho de peso que a dieta com promotores de crescimento.

Diante dos resultados obtidos, para uma melhor comprovação dos benefícios do óleo de aroeira, é necessário realizar estudos futuros realizando infecções experimentais nas aves e tratando-as com dietas que contenham a adição de óleo de aroeira.

Tabela 3 - Peso relativo dos Intestinos ( $\mathrm{g}_{100 \mathrm{~g}^{-1}}$ de peso vivo) de frangos de corte de 15 e de 21 dias, alimentados com dieta: sem antibiótico e sem anticoccidiano, com antibiótico e anticoccidiano, somente com antibiótico e com $0,4 \%$ de óleo de aroeira vermelha.

\begin{tabular}{lcc}
\hline & & \\
Dietas & $15^{\circ}$ dia de idade & 210 dia de idade \\
\hline Controle negativo (sem antibiótico e anticoccidiano) & $8,90 \mathrm{~A}$ & 6,56 \\
Controle positivo (com antibiótico e anticoccidiano) & $7,45 \mathrm{~B}$ & 6,18 \\
Somente com antibiótico & $7,58 \mathrm{~B}$ & 6,71 \\
$0,4 \%$ óleo de aroeira-vermelha & $7,30 \mathrm{~B}$ & 6,61 \\
\hline
\end{tabular}

\footnotetext{
${ }^{\mathrm{A}, \mathrm{B}}$ Médias seguidas por letras distintas na mesma coluna diferem significativamente pelo teste Tukey $(\mathrm{P}<0,05)$.
} 


\section{AGRADECIMENTOS}

Ao Conselho Nacional de Desenvolvimento Científico e Tecnológico (CNPq), pelo apoio financeiro concedido para execução desta pesquisa.

\section{COMITÊ DE ÉTICA E BIOSSEGURANÇA}

O protocolo de experimentação animal está de acordo com CONEA e foi aprovado pelo comitê de ética no uso de animais da Universidade Federal do Espírito Santo pelo protocolo n.01/08.

\section{REFERÊNCIAS}

ALVAREZ, L.C. et al. Evaluación de promotores del crecimiento para pollos de engorda. Veterinária México, v.24, n.2, p.141-144, 1994.

BALOG NETO, A. Efeito da utilização de simbiótico e do sistema de criação sobre o desempenho e morfometria do epitélio gastrintestinal de frangos de corte tipo colonial. Acta Scientiarum. Animal Sciences, v.29, n.4, p.379-385, 2007. Disponível em: <http://periodicos.uem.br/ojs/index.php/ ActaSciAnimSci/article/view/996/492>. Acesso em: $06 \mathrm{fev}$. 2011. doi: 10.4025/actascianimsci.v29i4.996.

BARBOSA, L.C.A. et al. Seasonal variation in the composition of volatile oils from Schinus terebinthifolius Raddi. Química Nova, v.30, n.8, p.1959-1965, 2007. Disponível em: <http:// www.scielo.br/pdf/qn/v30n8/a31v30n8.pdf>. Acesso em: $06 \mathrm{fev}$. 2011. doi: $10.1590 /$ S0100-40422007000800030.

BERTOLDI, M.C. Atividade antioxidante in vitro da fração fenólica, das oleorresinas e do óleo essencial de pimenta rosa (Schinus terebinthifolius Raddi). 2006. 96f. Dissertação (Mestrado em Ciência e Tecnologia de Alimentos) - Curso de Pós-graduação em Ciência e Tecnologia de Alimentos, Universidade Federal de Viçosa, MG.

CAVALCANTI, J.S. et al. Probióticos e farinha de carne e ossos com diversos níveis de contaminação bacteriana para frangos de corte. In: REUNIÃO ANUAL DA SOCIEDADE BRAZILEIRA DE ZOOTECNIA, 23., 1996, Fortaleza. Anais... Fortaleza: SBZ, 1996. p.50-52.

COATES, M.E. et al. A comparison of the growth of chicks in the Gustafsson germ-free apparatus and in a conventional environment with and without dietary supplement of penicillin. Brasilian Journal Nutrition, v.17, p.141-150, 1963.

CORNELI, J. Avaliação de promotores de crescimento alternativos em substituição aos convencionais sobre o desempenho, características de carcaça e morfometria intestinal em frangos de corte. 2004. 46f. Dissertação de Mestrado em Zootecnia - Curso de Pós-graduação em Zootecnia, Universidade Federal de Santa Maria, Santa Maria, RS.

CROMWELL, G.L. Antimicrobial agents. In: MILLER E. R. et al. Swine nutrition. Boston: Butterworth-Heinemann, 1991. p.297-314.

FREITAS, R. et al. Utilização do alho (Allium sativun) como promotor de crescimento em frangos de corte. Revista Brasileira de Zootecnia, v.30, n.3, p.761-765, 2001. Disponível em: <http:/ /www.scielo.br/pdf/rbz/v30n3/5245.pdf>. Acesso em: 06 fev. 2011. doi: $10.1590 / \mathrm{S} 1516-35982001000300022$.
FUKAYAMA, E.H. Extrato de orégano como aditivo em rações de frangos de corte. 2004. 61f. Dissertação de Mestrado em Zootecnia - Curso de Pós-graduação em Zootecnia, Universidade Federal de Lavras, Lavras, MG.

GAZZANEO, L.R.S. et al. Knowledge and use of medicinal plants by local specialists in a region of Atlantic Forest in the state of Pernambuco (Northeastern Brazil). Journal Ethnobiol Ethnomedicine, v.1, p.9, 2005. Disponível em: <http:// www.ncbi.nlm.nih.gov/pmc/articles/PMC1291389/>. Acesso em: 06 fev. 2011. doi: 10.1186/1746-4269-1-9.

HENRIQUE, A.P.F. et al. Uso de probióticos e antibióticos como promotores de crescimento para frangos de corte. In: REUNIÃO ANUAL DA SBZ, 2000, Viçosa, MG. Anais... Viçosa, MG: SBZ, 2000. 3p. 1 CD-ROM.

LIMA, I.O. et al. Atividade antifúngica de óleos essenciais sobre espécies de Candida. Revista Brasileira Farmacognosia, v.16, p.197-20, 2006a. Disponível em: <http://www.scielo.br/pdf/rbfar/v16n2/v16n2a11.pdf>. Acesso em: 06 fev. 2011. doi: 10.1590/S0102-695X2006000200011.

LIMA, M.R.F. et al. The antibiotic activity of some Brazilian medicinal plants. Revista Brasileira Farmacognosia, v.16, p.300-306, 2006b. Disponível em: <http://www.scielo.br/pdf/ rbfar/v16n3/a04v16n3.pdf>. Acesso em: 06 fev. 2011. doi: 10.1590/S0102-695X2006000300004.

LODDI, M.M. et al. Efect of the use of probiotic and antibiotic on the performance, yield and carcass quality of broilers. Revista Brasileira de Zootecnia, v.29, n.4, p. 1124-1131, 2000. Disponível em: <http://www.scielo.br/pdf/rbz/v29n4/ 5628.pdf >. Acesso em: 06 fev. 2011. doi: 10.1590/S151635982000000400025 .

LUQUETTI, B.C. Efeito da vacinação contra coccidiose aviária e da suplementação de glutamina ou prebiótico sobre a mucosa intestinal em frangos. 2005. 52f. Tese de Doutorado em Zootecnia - Faculdade de Ciências Agrárias e Veterinárias, Universidade Estadual Paulista, Jaboticabal, SP.

MACARI, M. et al. Fisiologia aplicada a frangos de corte: imunologia aplicada. Jaboticabal: FUNEP/UNESP, 1994. p. 231-245.

MAIORKA, A. et al. Influência da suplementação de glutamina sobre o desempenho e o desenvolvimento de vilos e criptas do intestino delgado de frangos. Arquivo Brasileiro de Medicina Veterinária e Zootecnia, v.52, n.5, p.487-490, 2000. Disponível em: <http:// www.scielo.br/scielo.php? script $=$ sci_arttext $\&$ pid $=S 0102$ 09352000000500014>. Acesso em: 06 fev. 2011. doi: 10.1590/ S0102-09352000000500014.

MARTINEZ, M.J. et al. Screening of some Cuban medicinal plants for antimicrobial activity. Journal Ethnopharmacol, v.52, p.171-174, 1996.

MELO JÚNIOR, E.J.M. et al. Estudo de plantas medicinais com atividade antimicrobiana sobre microrganismos presentes na alveolite. Revista ABO Nacional, v.8, n.4, p.220-226, 2000.

MENTEN, J.F.M. Aditivos alternativos na nutrição de aves: probióticos e prebióticos. In: REUNIÃO ANUAL DA SBZ, 38., 2001, Piracicaba. Anais... Piracicaba: SBZ, 2001. 1 CD-ROM. 
MENTEN, J.F.M. Probióticos, prebióticos e aditivos fitogênicos na nutrição de aves. In: SIMPÓSIO SOBRE INGREDIENTES NA ALIMENTAÇÃO ANIMAL, 2., 2002, Uberlândia, MG. Anais... Local: Uberlândia: CBNA, 2002. p.251-276.

MONTAGNE, L. et al. A review of interactions between dietary fibre and the intestinal mucosa, and their consequences on digestive health in young non-ruminant animals. Animal Feed Science and Technology, v.108, p.95-117, 2003

MOREIRA, J. et al. Efeito do uso de probiótico sobre o desempenho e rendimento de carcaça em frangos de corte. In: REUNIÃO ANUAL DA SBZ, 38., 2001, Piracicaba, SP. Anais... Piracicaba: SBZ, 2001. 1 CD-ROM.

POLLMAN, O.S. Probiotics in pig diets. In: RECENT ADVANCE IN ANIMAL NUTRITION, 1986. Proccedings... London: Haresign W and Cole DJA, 1986. p.193-205.
SCHMOURLO, G. et al. Screening of antifungal agents using ethanol precipitation and bioautography of medicinal and food plants. Journal Ethnopharmacol, v.96, n.3, p.563-568, 2005.

SILVA, M.A. et al. Intestinal mucosa structure of broiler chickens infected experimentally with Eimeria tenella and treated with essential oil of oregano. Ciência Rural, v.39, n.5, p.14711477, 2009. Disponível em: <http://www.scielo.br/pdf/cr/ v39n5/a212cr1049.pdf >. Acesso em: 06 fev. 2011. doi: 10.1590/S0103-84782009005000135.

TSINAS, A. C. Naturally selected. England, Meriden: Northampton, 2003. 80p.

UNIVERSIDADE FEDERAL DE VIÇOSA - UFV. Manual de utilização do Programa SAEG (Sistema para Análise Estatística e Genéticas). Viçosa, 1997. 150p. 